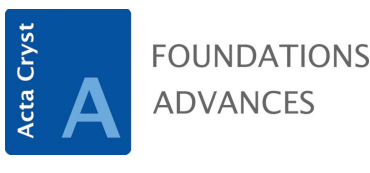

ISSN 2053-2733

Keywords: book review; Islamic geometric patterns.

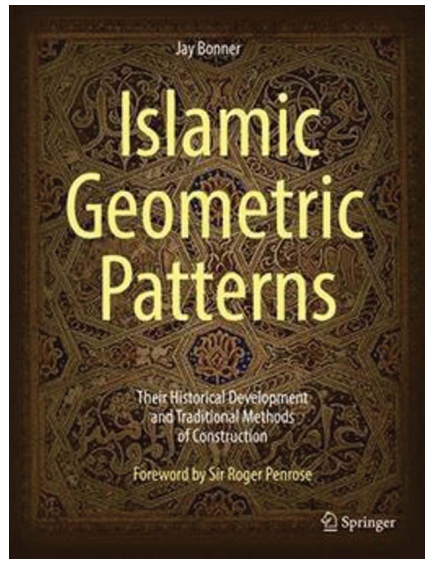

(C) 2019 International Union of Crystallography

\section{Islamic Geometric Patterns: Their Historical Development and Traditional Methods of Construction (with a chapter on the use of computer algorithms to generate Islamic geometric patterns by Craig Kaplan). By Jay Bonner. Springer, 2017. Pp. xxv+525. Price USD 139.00. ISBN 978-1-4939-7921-9 (softcover), 978-1- 4419-0216-0 (hardcover).}

\author{
Greg McColm*
}

Mathematics and Statistics, University of South Florida-Tampa, 4202 E. Fowler Avenue, PHY114, Tampa, Florida, 33620, USA. *Correspondence e-mail: mccolm@usf.edu

The popularity of geometric art of high symmetry has varied in time and space, and one of the high marks was in the medieval Middle East. The inspiration was probably ancient [pre-Islamic art in the Arabian subcontinent included textiles and calligraphy (Bloom \& Blair, 1997)], but it was during the Islamic expansion of the seventh and eighth centuries that Arabs encountered the architectural vegetal and geometric art of Mesopotamia, Greece and elsewhere. One product of the 'Islamic Golden Age' that spanned the eighth to 14th centuries was what Europeans later called 'arabesque' art.

Wichmann \& Wade (2017) divided this largely non-representational visual art into three classes, although it may be more of a spectrum than a classification. First of all, there is calligraphy. Second, there is vegetal arabesque, which had longstanding popularity in the Middle East prior to Islam. Third, there was geometric art, which was also popular prior to Islam, and which this book focuses on. See Figs. 1 and 2.

Most of the book focuses on a particularly sophisticated style of geometric art that flourished during the Golden Age and then, according to Bonner and other authors, was gradually displaced by different styles of arabesque art. Bonner does not ascribe a name to this style, which we might call 'geometric arabesque', and which consists of polygonal or curvilinear ribbons that weave into finite or indefinite geometric patterns. There were pre-Islamic precursors to this style (see Fig. 3), but it was during the Golden Age that this style ceased to be the ornament and became the show (see Fig. 4).

Several techniques have been advocated as methods used by artists to generate the geometric arabesque patterns in Golden Age art, and Bonner reviews several of them. But Bonner is an advocate for one of them, which he denotes the polygonal technique. One starts with a tessellation, and then using specific points on the polygon as a guide (such as midpoints of sides), one draws lines for, say, another tessellation, from which one obtains a mosaic. He exhibits an example described in an anonymous medieval source, On Similar and Complementary Interlocking Figures, for which he found one physical example (see Figs. 5, 6 and 7).

Bonner does review some other techniques that have been championed, but his focus is on the 'polygonal technique', which he argues lends itself more readily to innovation than its leading competitor, which he names the 'point-join' technique - although he does say that the latter technique, which also appears in the medieval literature, lends itself to copying extant patterns. He describes several other techniques as well.

The reason for this controversy over techniques is the lack of surviving medieval texts. Complicating this deficiency is the problem that '... scholarship of this vast subject remains underrepresented in two key areas: historical development and design methodology' (Bonner, p. ix). This book is intended to ' ... empower those with a sincere and dedicated interest in applying the creative processes of the past to their own original work' - and, presumably, to encourage more scholarship.

Returning to the polygonal technique, since medieval sources are scarce and architectural and other physical patterns more common, determining where a pattern came 


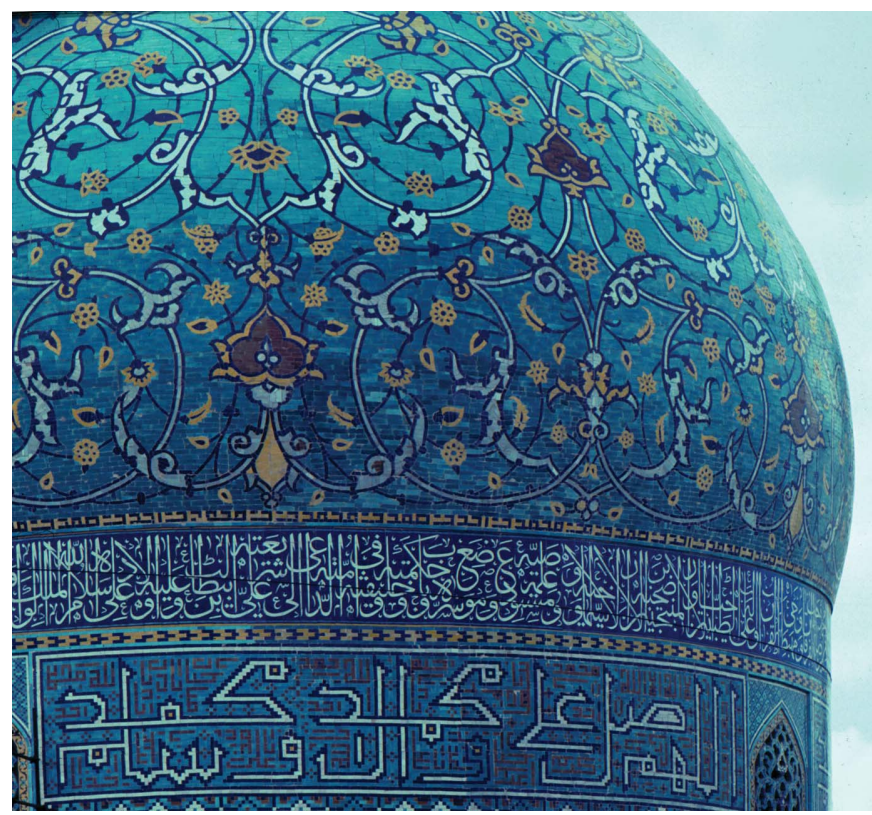

Figure 1

[Photograph 95 on page 140] The dome of the Mardar-i Shah madrasa in Isfahan, Iran. The design on the dome is a vegetal ('floral') arabesque, and the design on the dome's drum is calligraphic. Reproduced with permission from the Wade Archive at https://patterninislamicart.com/. Copyright David Wade.

from requires reverse engineering, and sometimes there are several possibilities. For example, the cover of an antique Qur'an from Baghdad could have been designed using at least two routes (see Figs. 8-11). Notice that the resulting cover could be described as calligraphic, vegetal and geometric, all at once. In this case, the polygonal technique - if indeed that is what the bookbinder used - generates ribbons that weave around the design.

This book is essentially a gigantic catalog of geometric arabesque designs, mostly from medieval or early modern

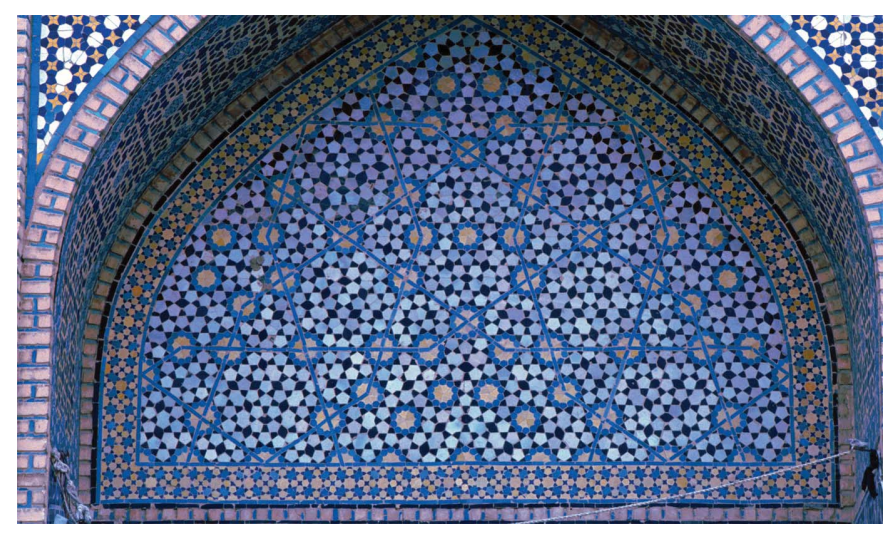

Figure 2

[Photograph 97 on page 142] A mosaic from the Hmamzada Darb-i Imam in Isfahan, Iran. Half of a ten-sided star occupies the lower center, and parts of three others at the apex and two sides; the rest of the pattern consists of kites, pentagons and non-convex octagons. Each of these large regions in turn consists of smaller simple tiles. Reproduced with permission from the Wade Archive at https://patterninislamicart.com/. Copyright David Wade.

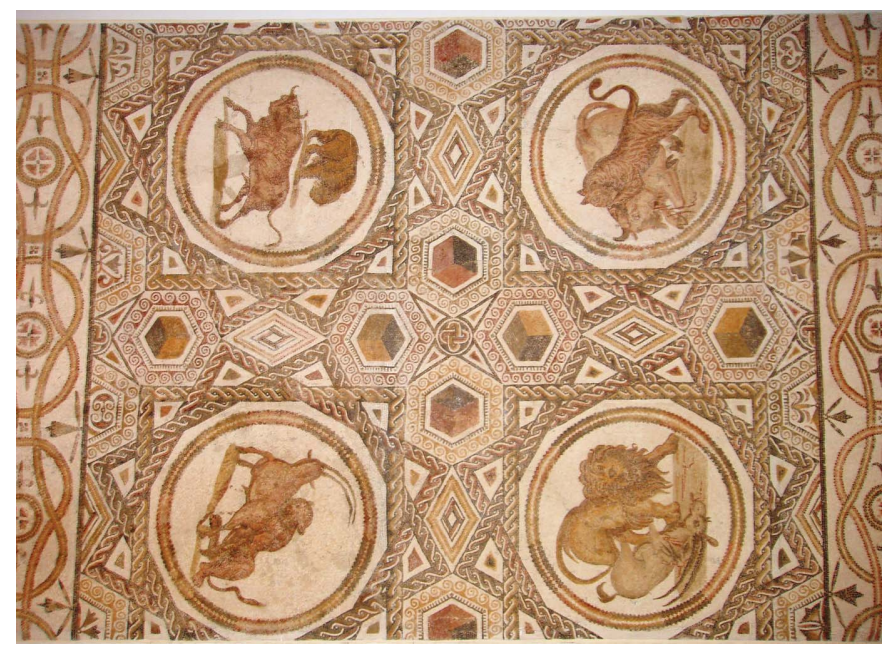

Figure 3

[Photograph 4 on page 13] Third century Roman mosaic. Like Italian Renaissance arabesque, the pattern is an ornament. Photograph courtesy of Damian Entwistle.

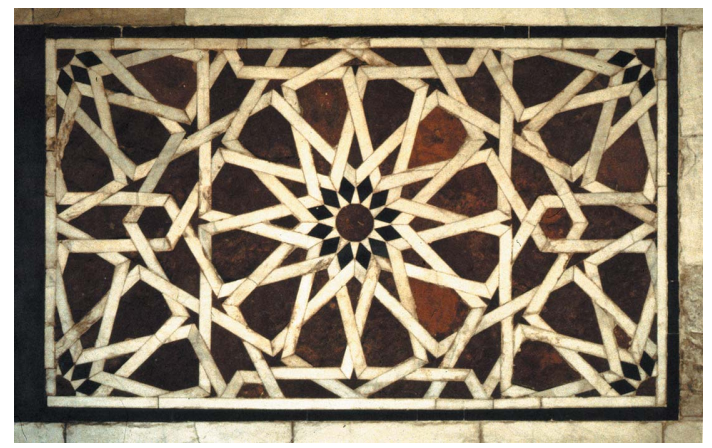

Figure 4

[Photograph 45 on page 87] Pattern from the Amir Aq Sunqar funerary complex in Cairo. The pattern is the art, not just an ornament. Notice that you can follow a ribbon from one end to the other. Reproduced with permission from the Wade Archive at https://patterninislamicart.com/. Copyright David Wade.

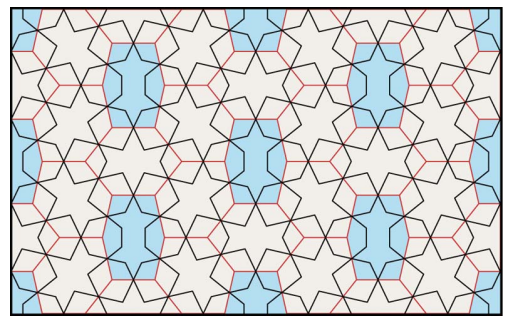

Figure 5

[Figure 57A on page 188] Start with a tessellation of the plane into three kinds of irregular convex hexagons. Then draw another tessellation with corners at midpoints of sides of the original tessellation. Reproduced with permission from Springer Nature. Copyright (2017) Springer Nature.

Islamic art, but with (comparatively) few photographs of physical examples. The patterns are primarily catalogued by some kind of local or underlying symmetry, often of the star shapes emerging from the ribbon. Thus we are introduced to two 'fourfold' systems, a fivefold system, a sevenfold system, and a variety of other systems, including an array of 'nonsys- 


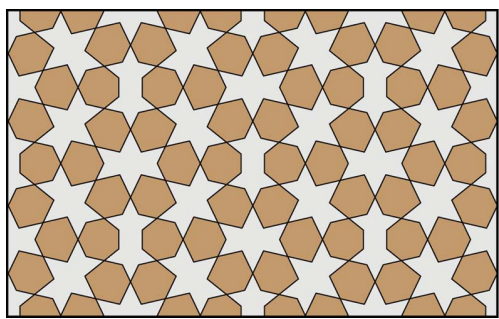

Figure 6

[Figure 57B on page 188] Remove the original tessellation and the result is a tessellation into one kind of irregular hexagon, one kind of nonconvex dodecagon, and one kind of non-convex tetradecagon. Reproduced with permission from Springer Nature. Copyright (2017) Springer Nature.

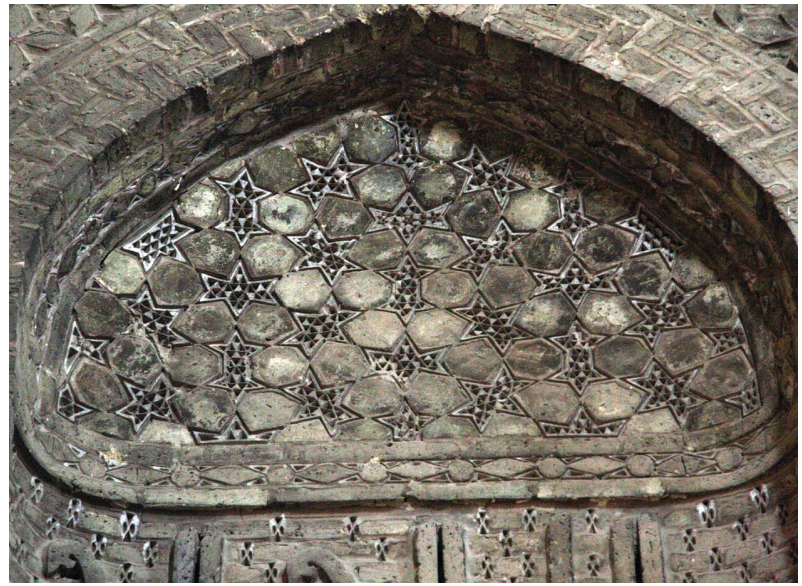

Figure 7

[Photograph 26 on page 44] A pattern from the Friday mosque in Isfahan, Iran. Copyright Tom Goris, reproduced with permission.

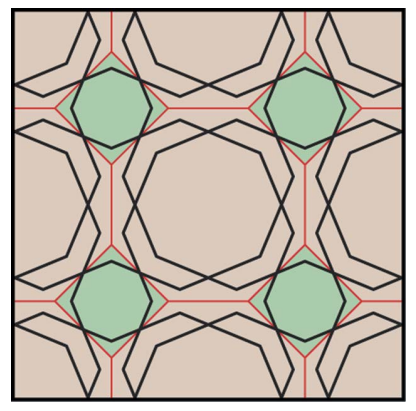

Figure 8

[Figure 127C on page 258] Start with a tessellation of regular octagons and squares. Draw the ribbon lines through the midpoints of the sides of the squares, and join them up without touching the other octagon sides. Reproduced with permission from Springer Nature. Copyright (2017) Springer Nature.

tematic' patterns. In the Foreword, Sir Roger Penrose suggested that the appearance of apparently non-crystallographic near-symmetries inspired some observers to propose that some of these patterns were quasicrystallographic. But careful examination of the polygonal reverse engineering of one beguiling example reveals an original crystallographic tessellation (see Fig. 12), and Bonner is generally skeptical about quasicrystalline patterns in Islamic art.

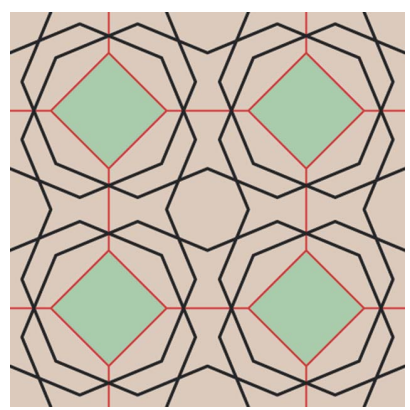

Figure 9

[Figure 128d on page 259] Alternatively, start with the same tessellation, but this time draw ribbon lines at two points of sides where two octagons meet. Notice that the resulting polygonal pattern is the same, if translated. Reproduced with permission from Springer Nature. Copyright (2017) Springer Nature.

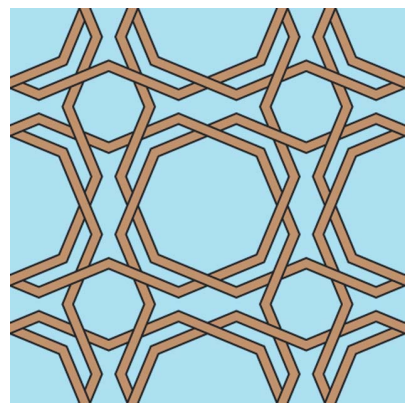

Figure 10

[Figure 127C on page 258] Either way, remove the octagons and squares and thicken the polygonal lines, determining the weave en route. Reproduced with permission from Springer Nature. Copyright (2017) Springer Nature.

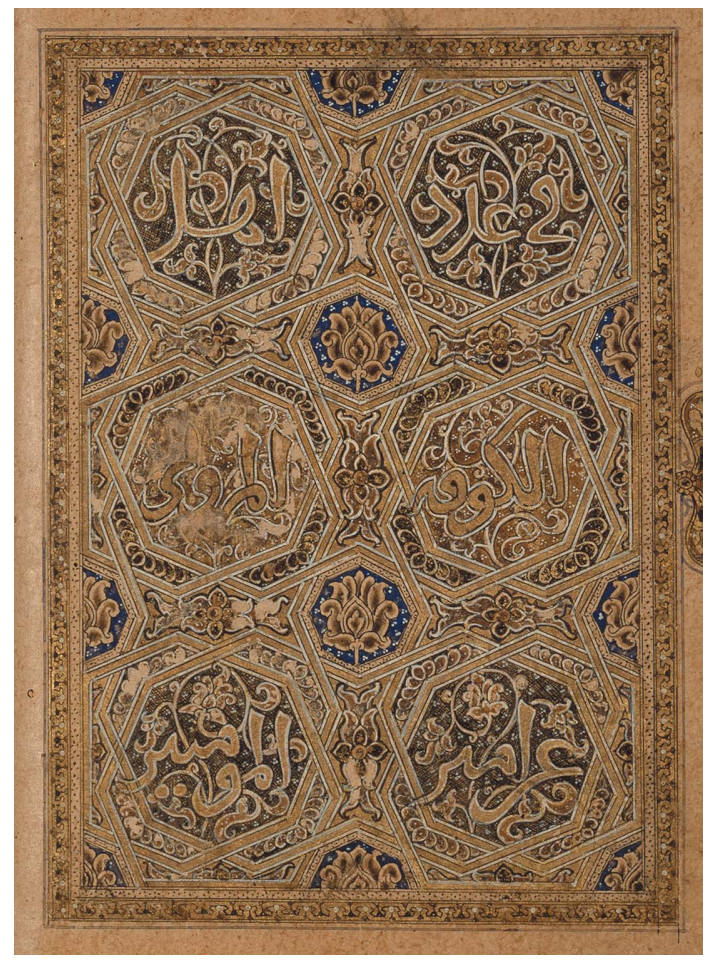

Figure 11

[Photograph 6 on page 17] Either way, here is the resulting cover. Notice the calligraphy and vegetal embellishment. Copyright The Trustees of the Chester Beatty Library, Dublin: CBL Is 1431, ff. 7b-8a. 


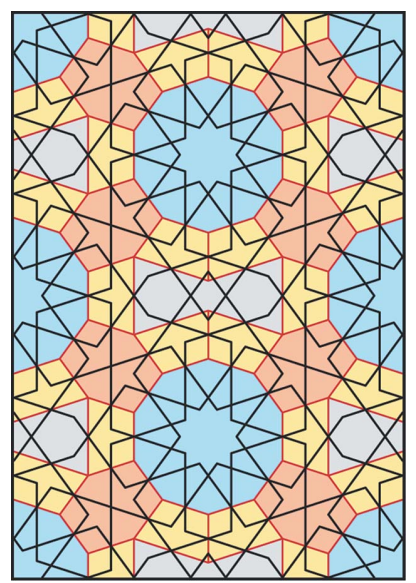

Figure 12

[Figure 223g on page 319] The ten-pointed stars arise from the decagons in the underlying tessellation, and this is an example of a 'fivefold' pattern. Nevertheless, this is crystallographic, of wallpaper group $\mathrm{cmm}$. Reproduced with permission from Springer Nature. Copyright (2017) Springer Nature.

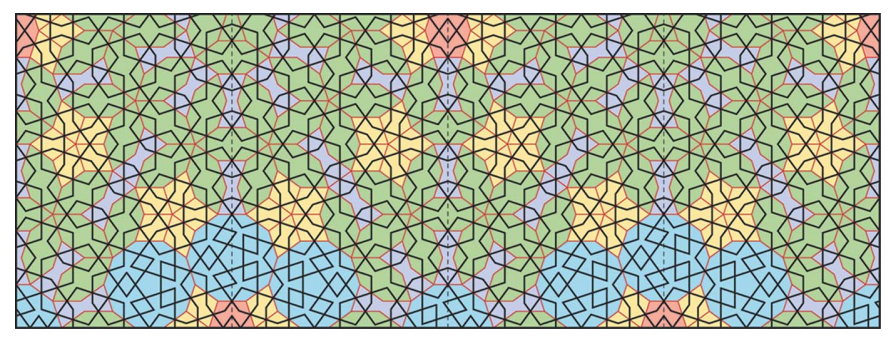

Figure 13

[Figure 240A on page 335] Start with a tessellation with numerous kinds of tiles and a large repeat unit. Draw ribbon lines through midpoints of appropriate sides. Reproduced with permission from Springer Nature. Copyright (2017) Springer Nature.

One reason for skepticism is a coincidence: the designs are finite - usually at most covering a panel or part of a wall while some of the most intriguing patterns have large unit cells. For example, Fig. 13 is of a fivefold pattern with a large repeat unit. This is the beginning of a complex pattern, for after generating the polygonal lines in Fig. 14, the artist then developed a lower-level pattern, which Bonner calls a 'duallevel' design. The resulting work is in Fig. 15.

One revelation from this book - one alluded to in the Preface - is how little we know. The historical record is thin: Baghdad itself was sacked by the Mongols in 1258, and the Golden Age gradually ended thereafter. Medieval Baghdad may well have been comparable to Renaissance Florence, but Baghdad did not leave us a comparably vast library of letters, books and official documents; all that went up in smoke in the 13th century. We may employ crystallographic groups to catalog and analyze medieval art, but that does not mean that the artists, their patrons or their public saw the art in the same

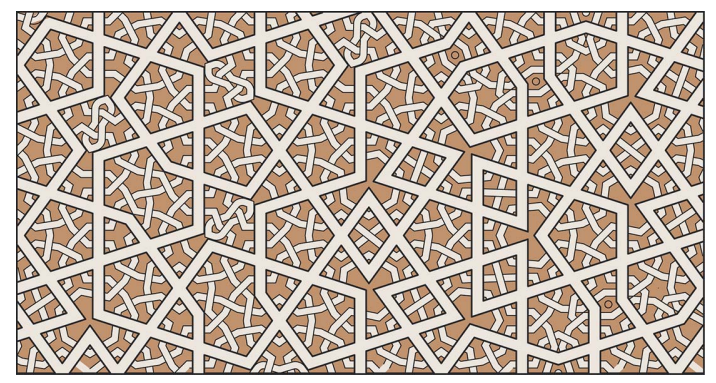

Figure 14

[Figure 67 on page 201] Remove the tessellation, and then add a finer array of polygonal lines by a similar process. Reproduced with permission from Springer Nature. Copyright (2017) Springer Nature.

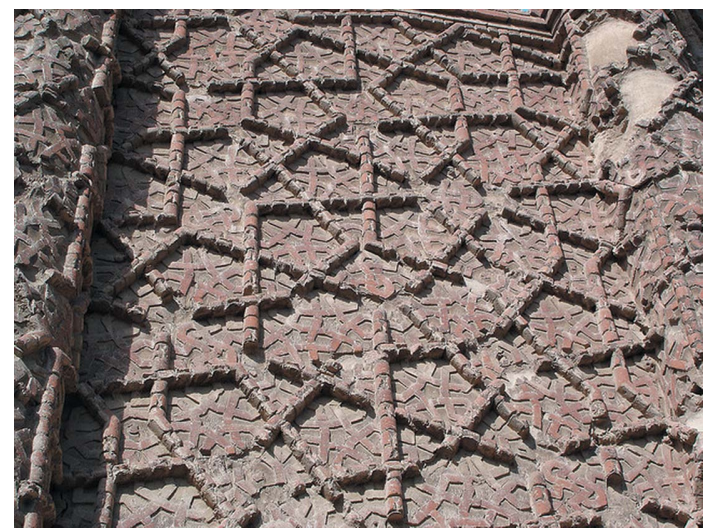

Figure 15

[Photograph 24 on page 42] From the façade of the Gunbad-i Qabud in Maragha, Iran. Copyright Richard Mortel, reproduced with permission.

way. Both the polygonal method and the point-joining method are suggestive of the local rules of modern tiling theory, but it is not clear whether there was a comparable theory, and if so, what it might have been.

This book is a place for Islamic scholars, artists and enthusiasts (and crystallographers mining the past for inspiration) to begin. That said, it is a somewhat difficult book; a reader unfamiliar with the geometry of Islamic art probably should read Chapter 2 before attempting Chapter 1. Both the glossary and the index are a bit thin. But to the extent that this book is an advertisement for the polygonal technique, the evidence Bonner amassed, however circumstantial, is considerable. And as a catalog, it certainly is a necessity for any institution with an Islamic art interest.

\section{References}

Bloom, J. \& Blair, S. (1997). Islamic Arts. London: Phaidon. Wichmann, B. \& Wade, D. (2017). Islamic Design: a Mathematical Approach. Cham: Birkhäuser. 